\title{
Multi-Level Balanced Isolated Floating Difference Amplifier
}

\author{
Yung-Cheng Tung, Shyr-Long Jeng, and Wei-Hua Chieng
}

\begin{abstract}
An innovative amplifier is proposed for applications involving high capacitive load and large voltage output swing, such as piezoelectric (PZT) actuator drivers. This amplifier is configured in a multi-level arrangement with floating amplifiers, yielding a high voltage gain as a sum of all individual gains from its operational amplifiers. The merits of such an amplifier also include a wide bandwidth and high potential power. Experiments using a six-level arrangement demonstrate a $100 \mathrm{kHz}$ bandwidth with $\pm 200 \mathrm{~V}$ output swing for different capacitive loadings.
\end{abstract}

Index Terms-Floating power supplies, piezoelectric (PZT) actuator.

\section{NOMENCLATURE}

\begin{tabular}{|c|c|}
\hline$\alpha, \alpha_{\perp}$ & Resistance ratio of closed-loop amplifier. \\
\hline$\alpha_{s}$ & Pre-scale ratio for input source voltage. \\
\hline$A_{D}, \mathbf{A}_{\mathbf{D}}$ & $\begin{array}{l}\text { Open loop dc gain and frequency response of } \\
\text { operational amplifier. }\end{array}$ \\
\hline $\mathbf{A}_{I}$ & Differential gain of isolation amplifier. \\
\hline$G(s), H(s)$ & Forward and feedback transfer function. \\
\hline$i, n$ & Number of level, also used in superscript. \\
\hline$j$ & Imaginary operator. \\
\hline$k$ & Equivalent loop gain. \\
\hline$s$ & Laplace operator. \\
\hline$T(s)$ & Transfer function of difference amplifier. \\
\hline$V_{i+}, V_{i-}$ & Input signals of difference amplifier. \\
\hline$V_{b}$ & Bias voltage on the power supply. \\
\hline$V_{b+}, V_{b-}$ & $\begin{array}{l}\text { Bias voltage on the power supply of syn- and } \\
\text { opposite- phase. }\end{array}$ \\
\hline$V_{b s}$ & Bias voltage of isolation amplifier. \\
\hline$V_{\text {in }}$ & Input voltage of isolation amplifier. \\
\hline$V_{o}$ & Output voltage of difference amplifier. \\
\hline$V_{o+}, V_{o-}$ & $\begin{array}{l}\text { Output voltage of difference amplifier for syn- } \\
\text { and opposite-phase. }\end{array}$ \\
\hline$V_{\text {out }}$ & Differential output of syn- and opposite-phase. \\
\hline$V_{p s}$ & Power supplies of operational amplifier. \\
\hline
\end{tabular}

Manuscript received December 6, 2005; revised October 21, 2007. First published April 18, 2008; current version published November 21, 2008. This work was supported by the National Science Council, R.O.C., under Contract NSC952218-E-009-002. This paper was recommended by Associate Editor I. Bell.

Y.-C. Tung and W.-H. Chieng are with Department of Mechanical Engineering, National Chiao-Tung University, Taiwan (e-mail: ken.me90g@nctu. edu.tw; whc@cc.nctu.edu.tw).

S.-L. Jeng is with Department of Automatic Engineering, Ta Hua Institute of Technology, Taiwan (e-mail: aets1@et4.thit.edu.tw).

Digital Object Identifier 10.1109/TCSI.2008.923283

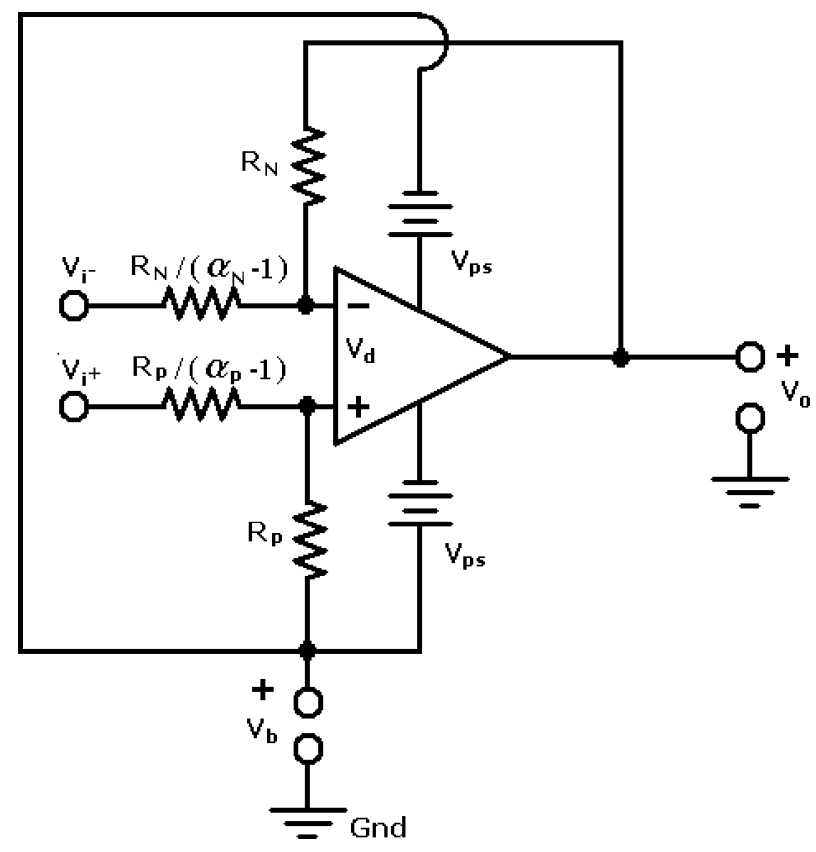

Fig. 1. Difference amplifier.

$\begin{array}{ll}V_{s} & \text { Input source voltage. } \\ \omega & \text { Frequency operator. } \\ \omega_{c} & \text { Cutoff frequency of operational amplifier. } \\ \omega_{c m} & \text { Bandwidth of MBIFDA. } \\ \omega_{c i}, \omega_{c s} & \text { Bandwidth of isolation and difference amplifier. } \\ \omega_{f} & \text { Bandwidth of the filter. }\end{array}$

\section{INTRODUCTION}

$\mathbf{V}$ ARIOUS circuits for driving capacitive loads have been presented in recent years, mostly focusing on the frequency compensation and the device fabrication in the domain of small signal/power applications [1]-[6]. Capacitive load driver circuits with particular application to piezoelectric actuators (PZTs) often requires a large output voltage swing of up to several hundred volts. Possible ways of driving PZT actuators include voltage supplies and charge pumps [7]-[9] with different characteristics [10]-[12].

Piezoelectric transducers exhibit less hysteresis when driven by current or charge than when driven by voltage [13]. As discussed in [13] and references therein, due to the uncontrolled nature of the output voltage, circuit offsets generally lead to the load capacitor being charged up. Saturation and distortion occur when the output voltage reaches the power supply rails. 
The stated complexity invariably refers to additional circuitry required to avoid charging of the capacitor. A popular method is to short the loading circuit, or periodically discharge the loading capacitance and reset the DC voltage to ground during handling. The resulting loads induce undesirable high-frequency disturbance, and significantly distort the control signal that is applied to the piezoelectric load. The noise generation is an undesirable attribute of most charge or current amplifiers. Unwanted noise can incur various problems.

The PZT actuator applied in precision machineries such as the impact drive mechanism (IDM) [14] requires a highly precise dynamic response. The driving circuit should account for also the wide operational bandwidth required in the feedback control loop. Huang [10] attempted to drive a PZT actuator using a battery charger. Their PWM-based system can only yield a step response time of slightly less than $4 \mathrm{~ms}$, since the frequency of their PWM switch is merely tens of kilohertz, which is not sufficient for controlling the PZT dynamics. Although the merchant amplifying instrument, which is a TREK 601C (TREK INC., America), meets a specification of output $\pm 500 \mathrm{~V}$, it has an operational bandwidth of only $10 \mathrm{kHz}$.

This work proposes an amplifier topology that provides greater flexibility and wider bandwidth than those that rely on high-voltage op-amps. This amplifying circuit presents a voltage gain of approximately 100 , an output swing of $\pm 200 \mathrm{~V}$ and an operational bandwidth of around $100 \mathrm{kHz}$. This circuit, which comprises floating isolation amplifiers and power amplifiers, can achieve a high dc gain and high output swing. Furthermore, the power is distributed averagely among individual sub-amplifiers, thus maximizing the total power output.

\section{DIFFERENTIAL AMPLIFIER}

The differential gain $\mathbf{A}_{\mathbf{D}}$ of the operational amplifier (opamp) is given by

$$
\mathbf{A}_{\mathbf{D}}=\frac{A_{D}}{1+\frac{j \omega}{\omega_{c}}}
$$

where $\omega_{c}$ denotes the $3 \mathrm{~dB}$ cutoff frequency. Providing a bias voltage on the power supply $V_{b}$, the output voltage of the difference amplifier, as illustrated in Fig. 1, is expressed as

$V_{o}=\left(\frac{\alpha_{N}}{\alpha_{P}}\left(\alpha_{P}-1\right) V_{i+}-\left(\alpha_{N}-1\right) V_{i-}\right) \frac{\mathbf{A}_{\mathbf{D}}}{\mathbf{A}_{\mathbf{D}}+\alpha_{N}}+V_{b}$.

The bandwidth of the difference amplifier $\omega_{c s}$ is derived from (2) for $A_{D} \gg \alpha_{N}$

$$
\omega_{c s}=\left(1+\left(\frac{A_{D}}{\alpha_{N}}\right)\right) \omega_{c} \approx \frac{A_{D} \omega_{c}}{\alpha_{N}} .
$$

If $V_{b}=0$, then the output voltage swing is bounded by the power supply, i.e., $\pm V_{p s}$. As illustrated in Fig. 2(a), the dotted line indicating the output signal is bounded by solid lines indicating power supply voltages. Conversely, the supply voltages may be varied, i.e., $V_{b}=V_{b}(t)$, to yield different output signals as indicated in Fig. 2(b). Such a power supply providing output voltage $V_{p s}+V_{b}(t)$ is called the floating power supply (FPS). An op-amp feedback application should always be restricted by a constant-gain-bandwidth product [15]. According to (3), the

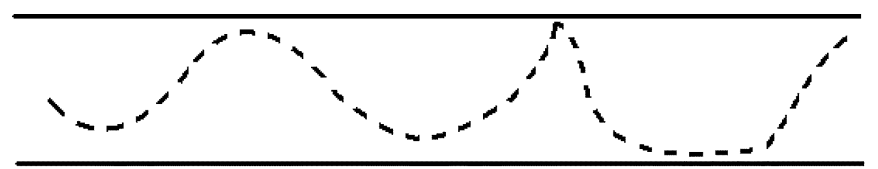

(a) $V_{b}=0$

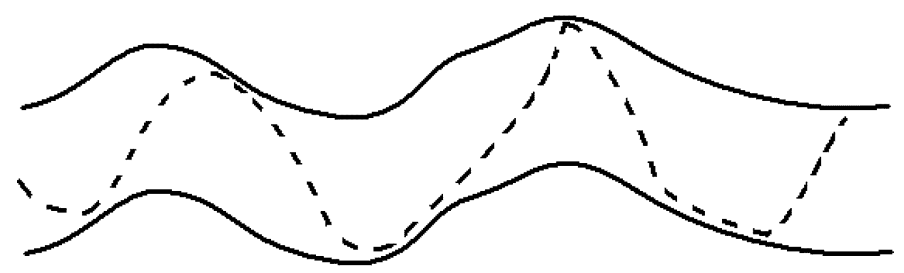

(b) $v_{b}=v_{b}(t)$

Fig. 2. Output signals from difference amplifier.

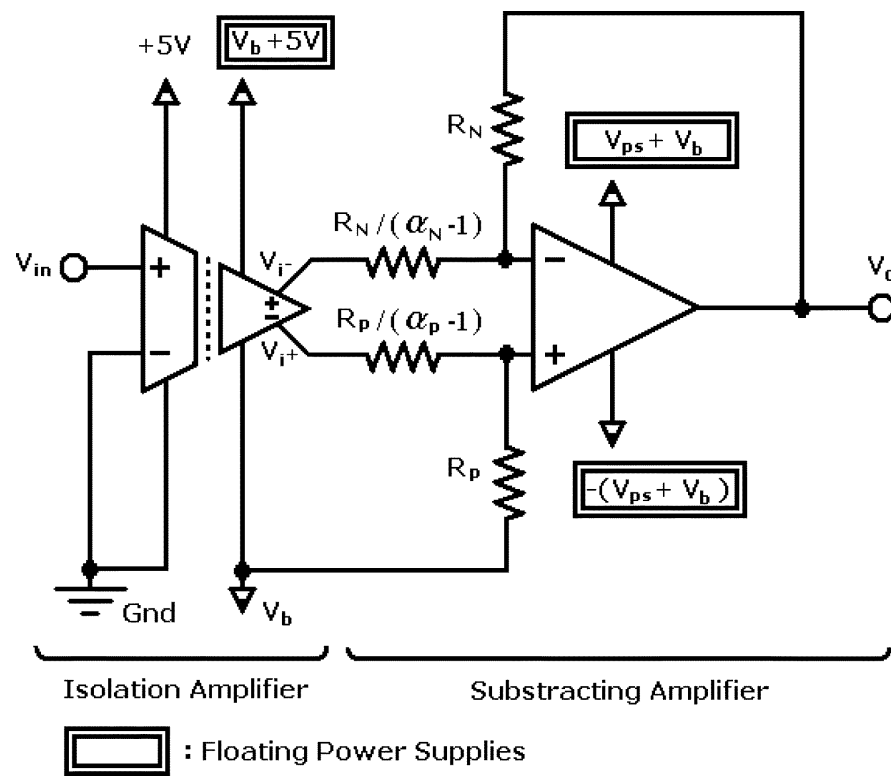

Fig. 3. Isolated floating difference amplifier (IFDA).

difference amplifier has a large close-loop bandwidth $\left(\omega_{c s}\right)$ corresponding to a small close-loop voltage gain $\left(\alpha_{n}\right)$. The restriction is in contrast to the PZT actuator application, which must satisfy simultaneously the requirements of wide bandwidth and large voltage gain.

\section{A. The Isolated Floating Difference Amplifier (IFDA)}

A difference amplifier using floating power supplies can generate sufficiently large output signal with respect to the ground as shown in Fig. 2(b). Such an amplifier structure may comprise an isolation amplifier, and a difference amplifier with floating power supplies called the isolated floating difference amplifier (IFDA), as illustrated in Fig. 3. Providing the differential gain of the isolation amplifier $\mathbf{A}_{\mathbf{I}}$ and the bias voltage of the isolation amplifier $V_{b s}$, the differential outputs of the isolation amplifier may be obtained where

$$
\begin{aligned}
& V_{i+}=-\frac{\mathbf{A}_{\mathbf{I}}}{2} V_{\text {in }}+V_{b}+V_{b s}, \text { and } \\
& V_{i-}=\frac{\mathbf{A}_{\mathbf{I}}}{2} V_{\text {in }}+V_{b}+V_{b s} .
\end{aligned}
$$

The isolation amplifier imposes a bias voltage $V_{b}$ on the input signal of difference amplifier. The biased input signal is then 


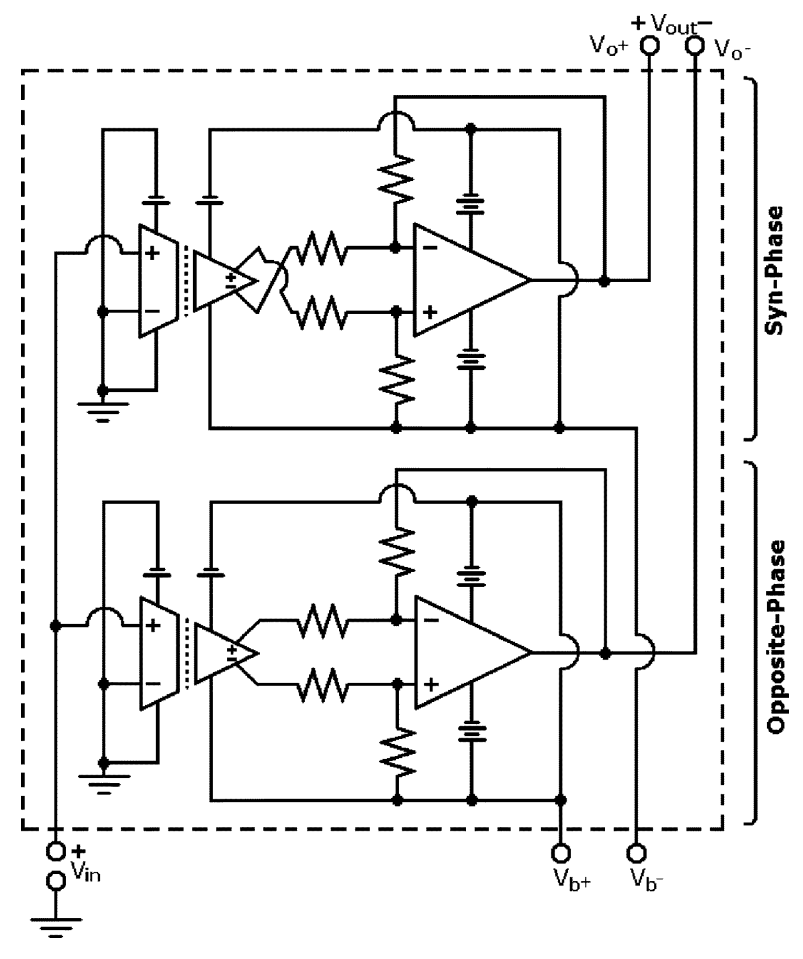

Fig. 4. Balanced isolated floating difference amplifier(BIFDA).

fed into the difference amplifier to modify the common-mode voltage. For simplicity, $\alpha_{P}-1=\alpha_{N}-1=\alpha$ may be selected, where $A_{D} \gg \alpha \gg 1$. By substituting (4a) and (4b) into (3), the output of an IFDA is obtained as

$$
V_{o}=-\frac{\mathbf{A}_{\mathbf{D}} \mathbf{A}_{\mathbf{I}} \alpha V_{\text {in }}}{\mathbf{A}_{\mathbf{D}}+\alpha_{N}}+V_{b} .
$$

\section{B. The Balanced Isolated Floating Difference Amplifier (BIFDA)}

Fig. 4 illustrates the proposed a balanced isolated floating difference amplifier (BIFDA) based on the isolated floating difference amplifier (IFDA). Each BIFDA comprises one syn-phase (noninverting) amplifier and one opposite-phase (inverting) amplifier. The balanced module employs three inputs and two outputs. The syn-phase and opposite-phase amplifier employs the same input $V_{\text {in }}$ but different biases, i.e., $V_{b+}$ and $V_{b-}$. The outputs of BIFDA determined from (5) as

$$
\begin{aligned}
& V_{o+}=\frac{\mathbf{A}_{\mathbf{D}} \mathbf{A}_{\mathbf{I}} \alpha V_{\mathrm{in}}}{\mathbf{A}_{\mathbf{D}}+\alpha_{N}}+V_{b+} \\
& V_{o-}=-\frac{\mathbf{A}_{\mathbf{D}} \mathbf{A}_{\mathbf{I}} \alpha V_{\mathrm{in}}}{\mathbf{A}_{\mathbf{D}}+\alpha_{N}}+V_{b-} .
\end{aligned}
$$

The voltage difference between the differential outputs is then determined as follows:

$$
V_{\text {out }}=V_{o+}-V_{o-}=\frac{2 \mathbf{A}_{\mathbf{D}} \mathbf{A}_{\mathbf{I}} \alpha}{\mathbf{A}_{\mathbf{D}}+\alpha_{N}} V_{\mathrm{in}}+\left(V_{b+}-V_{b-}\right) .
$$

\section{The Multi-Level Balanced Isolated Floating Difference Amplifier (MBIFDA)}

Fig. 5 illustrates a multilevel arrangement of the BIFDA. The output of the level- $(i-1)$ IFDA is fed into the bias voltage

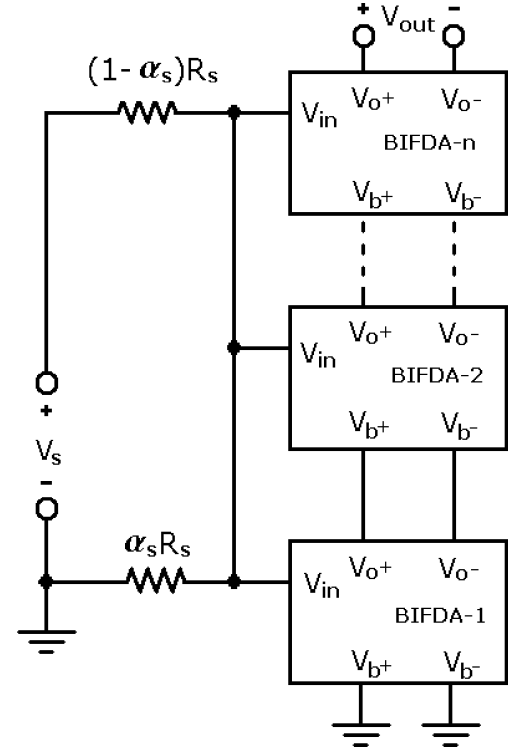

Fig. 5. Multi-level balanced isolated floating difference amplifier (MBIFSA).

input of the level- $i$ BIFDA. According to (7), the output voltage of level $i$ is given by

$$
V_{\text {out }}^{(i)}=2 \frac{\mathbf{A}_{\mathbf{D}} \mathbf{A}_{\mathbf{I}} \alpha}{\mathbf{A}_{\mathbf{D}}+\alpha_{N}} V_{\mathrm{in}}+V_{\text {out }}^{(i-1)} \text { for } i>2
$$

where the superscript $i$ denotes the level. The bias voltage inputs of the first level are connected to the ground. Therefore, the output from level $n$ is given by

$$
V_{\text {out }}=V_{\text {out }}^{(n)}=2 n \frac{\mathbf{A}_{\mathbf{D}} \mathbf{A}_{\mathbf{I}} \alpha}{\mathbf{A}_{\mathbf{D}}+\alpha_{N}} V_{\text {in }}=2 n \frac{\mathbf{A}_{\mathbf{D}} \mathbf{A}_{\mathbf{I}} \alpha \alpha_{s}}{\mathbf{A}_{\mathbf{D}}+\alpha_{N}} V_{s} .
$$

The 3-dB cutoff frequency (bandwidth) of the MBIFDA $\omega_{\mathrm{cm}}$ is obtained from (9),

$$
\omega_{c m}=\min \left(\omega_{c s}, \omega_{c i}\right)
$$

where $\omega_{c i}$ represents the bandwidth of $\mathbf{A}_{\mathbf{I}}$ of the isolation amplifier and is a constant value. Hence, it is reasonable to choose $\omega_{c m} \approx \omega_{c i}$.

\section{ANALYSIS OF CAPACTIVE LOADING}

Compared to (5), (9) depicts the additive relation of the outputs on each level of a MBIFDA. The analysis of MBIFDA can be simplified into a superposition of $2 n$ difference amplifiers. Fig. 6 illustrates the equivalent circuit of a difference amplifier with capacitive loading. In the circuit, identical capacitors $C_{I}$ are added to individual terminals of the difference amplifier to filter out the line noise. The transfer function described in Fig. 6 can be derived as

$$
T(S)=\frac{\left(1-\alpha_{N}\right)}{2} \frac{\mathrm{b}_{3} \mathrm{~s}^{3}+\mathrm{b}_{2} \mathrm{~s}^{2}+\mathrm{b}_{1} \mathrm{~s}+\mathrm{b}_{0}}{\mathrm{a}_{4} \mathrm{~s}^{4}+\mathrm{a}_{3} \mathrm{~s}^{3}+\mathrm{a}_{2} \mathrm{~s}^{2}+\mathrm{a}_{1} \mathrm{~s}+\mathrm{a}_{0}} .
$$




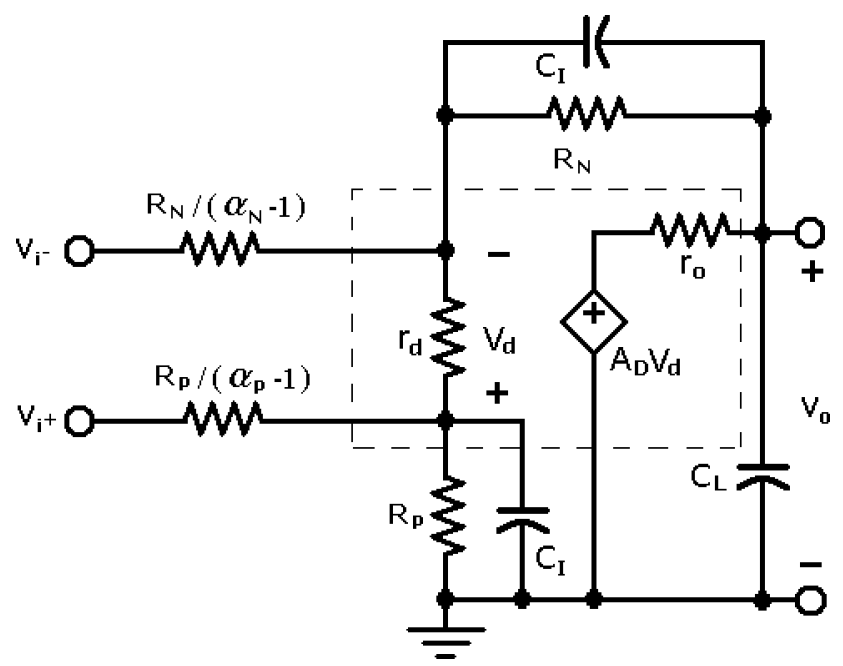

Fig. 6. Equivalent circuit of capacitance loading.

The result could be complicated. For simplicity, the following analysis is provided in the Appendix. The transfer function between the output and input voltage may be expressed as follows.

$$
T(s) \equiv \frac{V_{o}}{V_{\mathrm{in}}} \approx \frac{\alpha}{2}\left(\frac{k G(s)}{1+k G(s) H(s)}\right)
$$

where

$$
\begin{aligned}
k & =\frac{1}{C_{L}} \\
G(s) & =\frac{\left(2 \omega_{c s}-\frac{C_{I} r_{o} s^{2}}{\alpha_{N}}\right)}{r_{o} s^{2}\left(\frac{s}{\left(\alpha_{N} \omega_{f}\right)}+1\right)} \\
G(s) H(s) & =\frac{s\left(\frac{s}{\left(\alpha_{N} \omega_{f}\right)}+1\right)^{2}+\omega_{c s}}{r_{o} s^{2}\left(\frac{s}{\left(\alpha_{N} \omega_{f}\right)}+1\right)^{2}} .
\end{aligned}
$$

The above transfer function yields four poles and three zeros. The effect of $C_{L}$ and is discussed in Section IV.

\section{RESULTS AND COMPARISONS}

The power amplifier adopted in this work is LM-4700 (National Semiconductor Corp.). Following LM-4700 specification, the open loop voltage gain is $A_{D}=110 \mathrm{~dB}$, and gain-bandwidth product is typically $7.5 \mathrm{MHz}$. By observing the open loop frequency response given in the datasheet and determined from the gain-bandwidth product, the 3-dB cutoff frequency is obtained as $\omega_{c}=144.5 \mathrm{rad} / \mathrm{s}$. The maximum output power that can be delivered into the load is $30 \mathrm{~W}$. The isolation amplifier is HCPL-7800 (Agilent Technologies, Inc), which adopts the dc gain $\mathrm{A}_{I}=8$ and a bandwidth $\omega_{c i} \sim 628.3 \times 10^{3} \mathrm{rad} / \mathrm{s}$. The difference amplifier adopts the specification of $\mathrm{R}=22 \mathrm{k} \Omega, \alpha=11\left(\alpha_{N}=12\right)$, and $C_{I}=22 \mathrm{pF}$. Equation (3) confirms that the bandwidth of the difference amplifier is $\omega_{c s}=3.926 \times 10^{6} \mathrm{rad} / \mathrm{s}$. Additionally, (10) demonstrates that the bandwidth $\omega_{c m}$ of the MBIFDA is

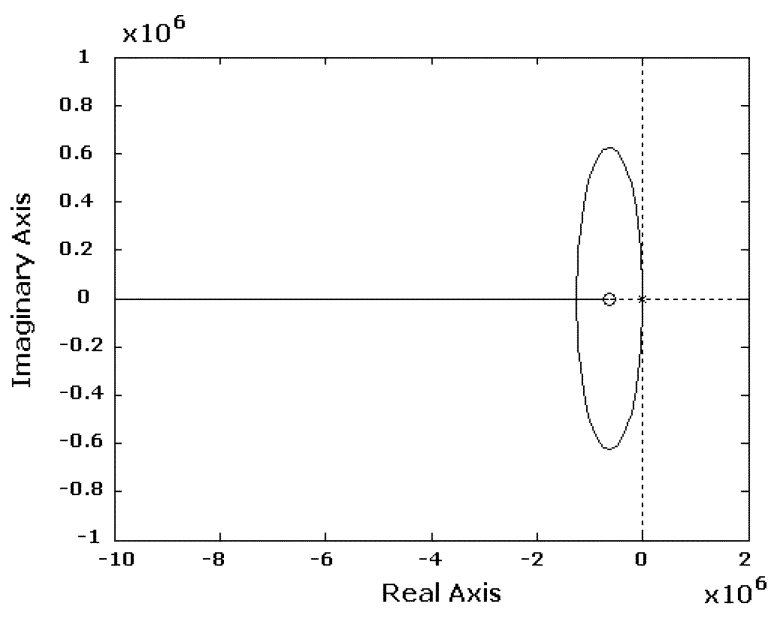

(a)

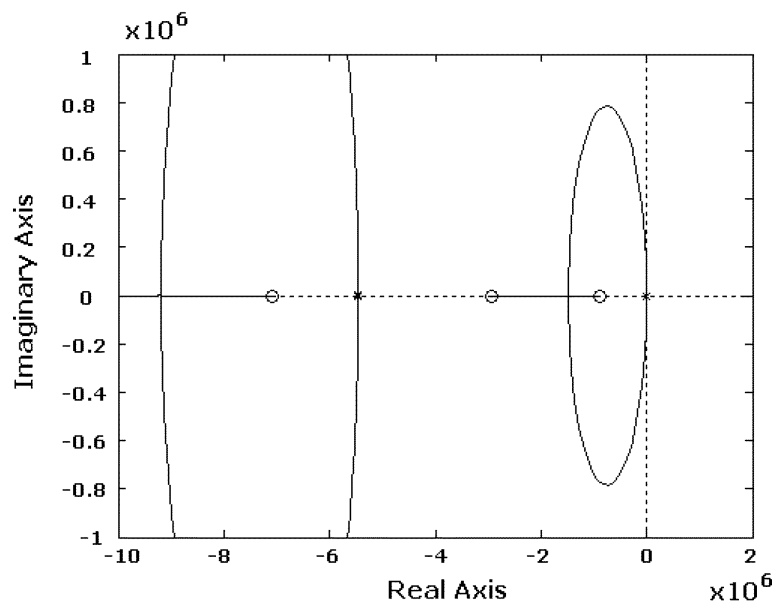

(b)

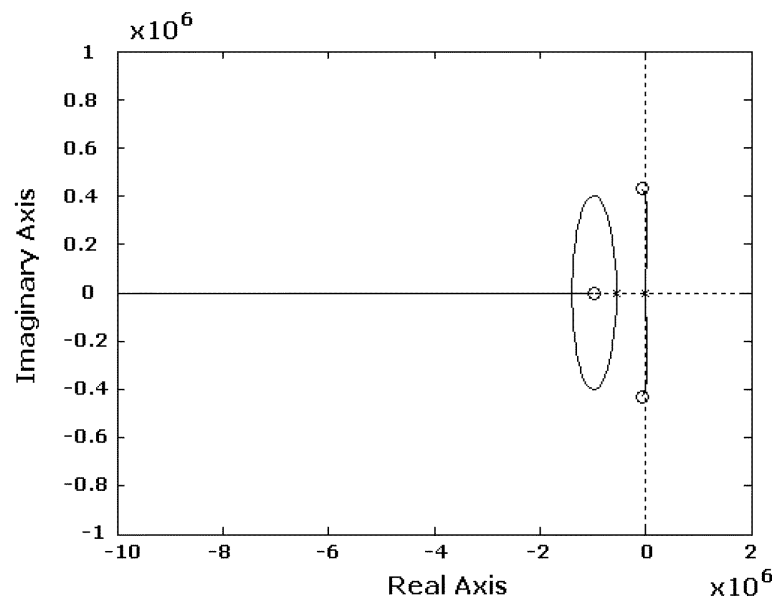

(c)

Fig. 7. Matlab ${ }^{\circledR}$ simulation: Root locus of capacitive loading (a) $\mathrm{C}_{I}=0 \mathrm{pF}$ (b) $\mathrm{C}_{I}=100 \mathrm{pF}$ (c) $\mathrm{C}_{I}=1000 \mathrm{pF}$.

$628.3 \times 10^{3} \mathrm{rad} / \mathrm{s}(100 \mathrm{kHz})$. Six levels, i.e., $n=6$, are used to implement the MBIFDA, and $\alpha_{s}=1 / 11$.

\section{A. Matlab Simulation for the Capacitive Loading}

Equation (12) can conveniently be adopted to verify the frequency response for different capacitive loadings. Fig. 7 illustrates the root locus of $T(s)$ for different $C_{L}$. Fig. 7(a) 


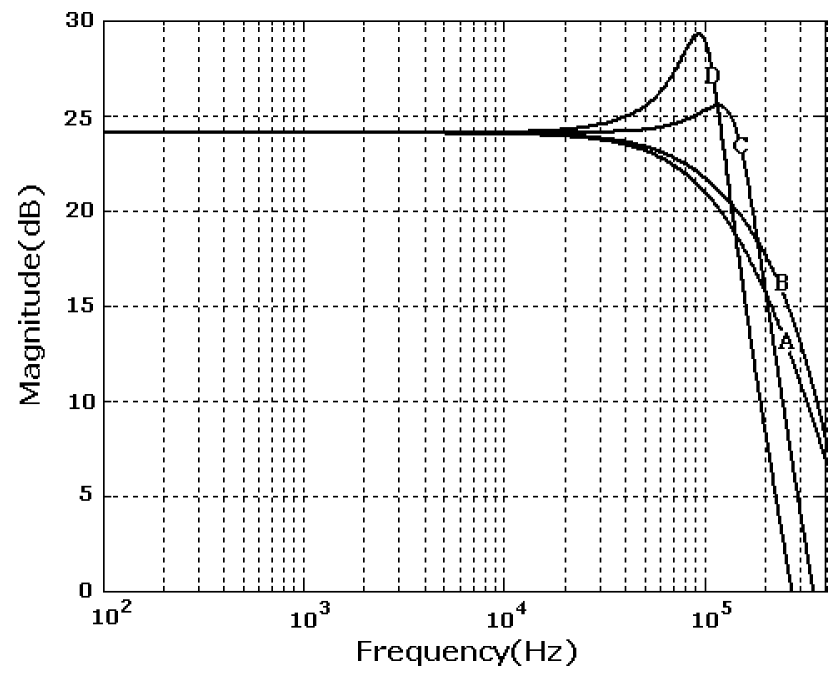

Fig. 8. Matlab ${ }^{\circledR}$ simulation: Frequency responses resulting from different capacitive loadings (a) $\mathrm{C}_{L}=10 \mathrm{pF}$, (b) $\mathrm{C}_{L}=10 \mathrm{nF}$, (c) $\mathrm{C}_{L}=50 \mathrm{nF}$, and (d) $\mathrm{C}_{L}=10^{2} \mathrm{nF}$.

illustrates the closed loop poles of the transfer function, which tend to approach the imaginary axis as $C_{L}$ increases (or $k$ decreases). Thus, the system becomes oscillating with large capacitive loading. Fig. 7(b) indicates that the root locus can be pulled away from the imaginary axis when a nonzero capacitance $C_{I}$ is introduced into the difference amplifier. However, a larger $C_{I}$ eventually destabilizes the system, as indicated in Fig. 7(c), where the filter capacitance $C_{I}$ not only filters the signal, but also play a critical rule in maintaining the stability of the system. Fig. 8 illustrates the frequency response using the aforementioned specification $C_{I}=22 \mathrm{pF}$. In Fig. 8, the bandwidth decreases as the capacitive loading $C_{L}$ increases, which is consistent with Fig. 7, where the dominant poles approach the imaginary axis as $C_{L}$ increases.

\section{B. Experimental Results}

Fig. 9(a) illustrates the experiment setup, which comprises a function generator, an oscilloscope and a differential probe to measure the frequency response. Fig. 9(b) illustrates the six outputs on individual levels of the MBIFSA with respect to the given sinusoidal inputs.

Fig. 10 illustrates the experimental results of outputs on individual levels of MBIFDA derive from the no-load test. The dc gains from the experiments are almost identical to the theoretical values obtained from (8).

$$
\frac{\left|V_{\text {out }}{ }^{(i)}\right|}{\left|V_{s}\right|}=2 i \frac{\mathrm{A}_{D} \mathrm{~A}_{I}}{\mathrm{~A}_{D}+\alpha_{N}} \approx 2 i \mathrm{~A}_{I} .
$$

The dc gains at level $i$ are obtained from (13) as $(24+$ $\left.20 \log _{10} i\right) \mathrm{dB}$. The bandwidth $(\sim 109 \mathrm{kHz})$ from the experiment is varied by $9 \%$ compared to the theoretical bandwidth $\omega_{c m}=628.3 \times 10^{3} \mathrm{rad} / \mathrm{s}(100 \mathrm{kHz})$.

Fig. 11 illustrates the experimental results for different capacitive loadings. The experiments results yield larger resonance peak values and narrower bandwidths than the simulation result, implying that the dominant poles of the MBIFDA occurred in the experiment are closer to the imaginary axis than that of

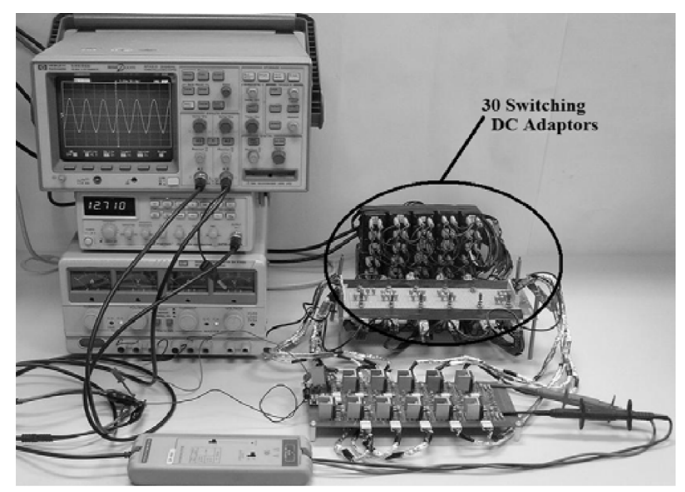

(a)

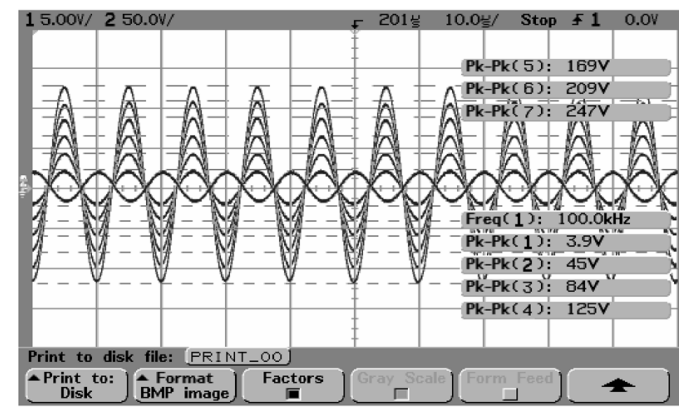

(b)

Fig. 9. Experiment: (a) photograph of experiment setup, and (b) result of $100 \mathrm{kHz}$ input/output on the oscilloscope.

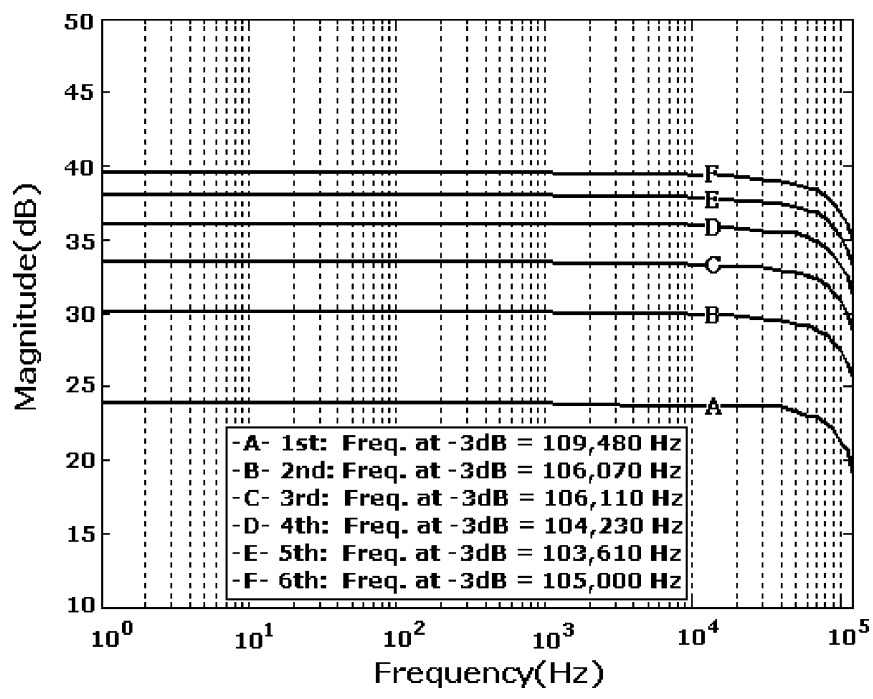

Fig. 10. Experimental Result: outputs on individual levels of MBIFDA due to the no-load test.

the theoretical result in (12). Such differences may result from the inaccuracy of the resistance and capacitance utilized in the experiment, and the differential probe poses a capacitance of nearly $10 \mathrm{pF}$. These experiments using a six-level arrangement show a bandwidth of $100 \mathrm{kHz}$ with an output swing of around $\pm 200 \mathrm{~V}$.

\section{CONCLUSION}

This work presents a multilevel balanced isolated floating difference amplifier (MBIFDA). Such an amplifier is appropriate for applications with high capacitive load and large 


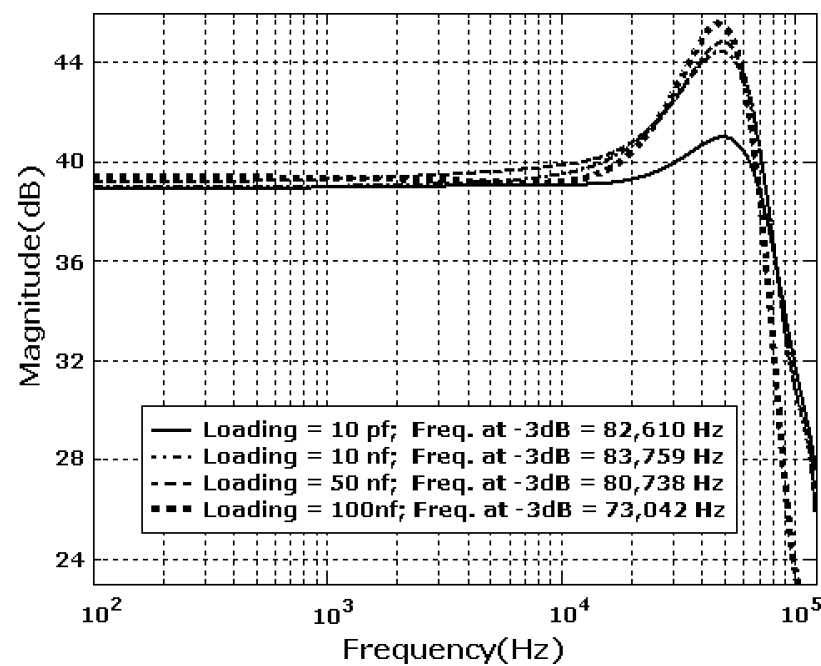

Fig. 11. Experimental Result: frequency responses due to difference capacitive loadings (a) $\mathrm{C}_{L}=10 \mathrm{pF}$, (b) $\mathrm{C}_{L}=10 \mathrm{nF}$, (c) $\mathrm{C}_{L}=50 \mathrm{nF}$, and (d) $\mathrm{C}_{L}=$ $10^{2} \mathrm{nF}$.

voltage output swing, such as PZT actuator drivers. Both the theoretical and experimental results indicate that the MBIFDA can sum up all gains in individual difference amplifiers, and the bandwidth is not sacrificed or decreased as in typical operational circuits. Thus the MBIFDA, as a voltage amplifier, transcends the principle of constant gain-bandwidth- product in the applications of operational amplifiers. Nevertheless, the circuitry is additive, and can be adopted in different voltage amplification purposes.

\section{APPENDIX}

Fig. 6 indicates that rearranging terms in Kirchhoff's Current Law (KCL) equations in each node yield a transfer function as

$$
T(S)=\frac{\left(1-\alpha_{N}\right)}{2} \frac{\mathrm{b}_{3} \mathrm{~s}^{3}+\mathrm{b}_{2} \mathrm{~s}^{2}+\mathrm{b}_{1} \mathrm{~s}+\mathrm{b}_{0}}{\mathrm{a}_{4} \mathrm{~s}^{4}+\mathrm{a}_{3} \mathrm{~s}^{3}+\mathrm{a}_{2} \mathrm{~s}^{2}+\mathrm{a}_{1} \mathrm{~s}+\mathrm{a}_{0}},
$$

assuming $\alpha_{P}=\alpha_{N}$ and $R_{P}=R_{N}$. Then

$$
\begin{aligned}
b_{3}= & r_{d} C_{I}^{2} R_{N}^{2} r_{o} \\
b_{2}= & r_{d}\left(C_{I} R_{N} r_{o}+\alpha_{N} C_{I} R_{N} r_{o}+C_{I}^{2} R_{N}^{2} r_{o} \omega_{c}\right) \\
b_{1}= & r_{d}\left(\alpha_{N} r_{o}-2 A_{D} C_{I} R_{N}^{2} \omega_{c}\right. \\
& \left.+C_{I} R_{N} r_{o} \omega_{c}+\alpha_{N} C_{I} R_{N} r_{o} \omega_{c}\right) \\
b_{0}= & r_{d}\left(-2 \alpha_{N} A_{D} R_{N} \omega_{c}+\alpha_{N} r_{o} \omega_{c}\right) \\
a_{4}= & C_{I}^{2} C_{L} R_{N}^{3} r_{d} r_{o} \\
a_{3}= & C_{I}\left(C_{I}+2 C_{L}\right) R_{N}^{3} r_{o}+C_{I}^{2} R_{N}^{3} r_{d}-C_{I}^{2} R_{N}^{2} r_{d} r_{o} \\
& +\alpha_{N} C_{I}^{2} R_{N}^{2} r_{d} r_{o}+2 \alpha_{N} C_{I} C_{L} R_{N}^{2} r_{d} r_{o} \\
& +C_{I}^{2} C_{L} R_{N}^{3} r_{d} r_{o} \omega_{c} \\
a_{2}= & C_{I} R_{N}^{3}+2 \alpha_{N} R_{N}^{2}\left(C_{I} r_{o}+C_{L} r_{o}+C_{I} r_{d}\right) \\
& +C_{I}\left(C_{I}+2 C_{L}\right) R_{N}^{3} r_{o} \omega_{c}-C_{I} R_{N} r_{d} r_{o} \\
& +\alpha_{N}^{2}\left(C_{I}+C_{L}\right) R_{N} r_{d} r_{o}+2 \alpha_{N} C_{I} C_{L} R_{N}^{2} r_{d} r_{o} \omega_{c} \\
& +A_{D} C_{I}^{2} R_{N}^{3} r_{d} \omega_{c}-C_{I}^{2} R_{N}^{2} r_{d} r_{o} \omega_{c}+\alpha_{N} C_{I}^{2} R_{N}^{2} r_{d} r_{o} \omega_{c} \\
& +C_{I}^{2} R_{N}^{3} r_{d} \omega_{c}
\end{aligned}
$$

$$
\begin{aligned}
a_{1}= & 2 \alpha_{N} R_{N}^{2}-R_{N} r_{o}+2 \alpha_{N} R_{N} r_{o}+\alpha_{N}^{2} R_{N} r_{d}-\alpha_{N} r_{d} r_{o} \\
& +\alpha_{N}^{2} r_{d} r_{o}+2 C_{I} R_{N}^{3} \omega_{c}+2 \alpha_{N} C_{I} R_{N}^{2} r_{o} \omega_{c} \\
& +2 \alpha_{N} C_{L} R_{N}^{2} r_{o} \omega_{c}+A_{D} C_{I} R_{N}^{2} r_{d} \omega_{c} \\
& +\alpha_{N}\left(2+A_{D}\right) C_{I} R_{N}^{2} r_{d} \omega_{c}-C_{I} R_{N} r_{d} r_{o} \omega_{c} \\
& +\alpha_{N}^{2}\left(C_{I}+C_{L}\right) R_{N} r_{d} r_{o} \omega_{c} \\
a_{0}= & 2 \alpha_{N} R_{N}^{2} \omega_{c}-R_{N} r_{o} \omega_{c}+2 \alpha_{N} R_{N} r_{o} \omega_{c}+\alpha_{N}^{2} R_{N} r_{d} \omega_{b} \\
& +\alpha_{N} A_{D} R_{N} r_{d} \omega_{c}-\alpha_{N} r_{d} r_{o} \omega_{c}+\alpha_{N}^{2} r_{d} r_{o} \omega_{c}
\end{aligned}
$$

Then, set $\omega_{f}=1 / C_{I} R_{N}$, and follow the conditions:

1) $\alpha_{N}>>1, \alpha_{N}>>\omega_{c} / \omega_{f}$,

$$
\Rightarrow b_{2} \approx r_{d} \alpha_{N} C_{I} R_{N} r_{o} \text {. }
$$

2) $1>>\omega_{c} / \omega_{f}, A_{D} \omega_{c}>>\omega_{f}>>\alpha_{N}, R_{N}>>r_{o}$,

$$
\Rightarrow b_{1} \approx-2 r_{d} A_{D} C_{I} R_{N}^{2} \omega_{c} \text {. }
$$

3) $A_{D}>>1, R_{N}>>r_{o}$

$$
\Rightarrow b_{0} \approx-2 r_{d} \alpha_{N} A_{D} R_{N} \omega_{c} .
$$

4) $\alpha_{N}>>1>>\omega_{c} / \omega_{f}, r_{d}>>R_{N}>>\alpha_{N} r_{o}$,

$$
\Rightarrow a_{3} \approx 2 \alpha_{N} C_{I} C_{L} R_{N}^{2} r_{d} r_{o}+C_{I}^{2} R_{N}^{3} r_{d} .
$$

5) $A_{D}>>2 \alpha_{N}>>1>A_{D} \omega_{c} / \omega_{f}, r_{d}>>R_{N}>>$ $\alpha_{N} r_{o}>>1, C_{I} r_{d}>>C_{L} r_{o}$,

$$
\Rightarrow a_{2} \approx \alpha_{N}^{2} C_{L} R_{N} r_{d} r_{o}+2 \alpha_{N} R_{N}^{2} C_{I} r_{d} .
$$

6) $A_{D}>>2 \alpha_{N}>>1>>A_{D} \omega_{c} / \omega_{f}, r_{d}>>R_{N}>>$ $\alpha_{N} r_{o}>>1,1 / \omega_{c}>>C_{L} r_{o}$,

$$
\Rightarrow a_{1} \approx \alpha_{N}^{2} R_{N} r_{d}
$$

7) $A_{D}>>\alpha_{N}>>1, r_{d}>>R_{N}>>r_{o}$,

$$
\Rightarrow a_{0} \approx \alpha_{N} A_{D} R_{N} r_{d} \omega_{c}
$$

\section{REFERENCES}

[1] F. Moraveji, "A tiny, high-speed, wide-band, voltage-feed-back amplifier stable with all capacitive load," IEEE J. Solid-State Circuits, vol. 31, no. 10, pp. 1511-1516, Oct. 1996.

[2] P. K. Chan and Y.C. Chen, "Gain-enhanced feedforward path compensation technique for pole-zero cancellation at heavy capacitive loads," IEEE Trans. Circuits Syst. II, Anal. Digit. Signal Process., vol. 50, no. 12, pp. 933-941, Dec. 2003.

[3] K. N. Leung, P. K. T. Mok, W.-H. Ki, and J. K. O. Sin, "Three stage large capacitive load amplifier with damping-factor control frequency compensation," IEEE Trans. Solid-State Circuits, vol. 35, no. 2, pp. 221-230, Feb. 2000.

[4] J. H. Lou and J. B. Kuo, "A 1.5-V full-swing bootstrapped CMOS large capacitive-load driver circuit suitable for low-voltage CMOS VLSI," IEEE J. Solid-State Circuits, vol. 32, no. 1, pp. 119-121, Jan. 1997.

[5] H. Parzhuber and W. Steinhagen, "An adaptive biasing one-stage CMOS operational amplifier for driving high capacitive loads," IEEE, J. Solid-State Circuits, vol. 26, no. 10, pp. 1457-1460, Oct. 1991.

[6] A. Nosratinia, M. Ahmadi, G. A. Jullien, and M. Shridhar, "A highdrive CMOS buffer for high capacitive loads," in Int. Conf. on Circuits Syst., Shenzhen, China, Jun. 1991, pp. 648-650.

[7] G. S. Choi, H. S. Kim, and G. H. Choi, "A study on position control piezoelectric actuators," in Proc. ISIE, 1997, pp. 851-855. 
[8] C. Saas, A. Wroblewski, and J. A. Nossek, "Low-power DA-converters for display applications using stepwise charging and charge recovery," Proc. IEEE ISCAS' 04, vol. 2, p. II-277-20, May 2004.

[9] G. Palumbo and D. Pappalardo, "Charge pump circuits with only capacitive loads: Optimized design," IEEE Trans. Circuits Syst. II, Expr. Briefs, vol. 53, no. 2, pp. 128-132, Feb. 2006.

[10] Y. C. Huang, W. Y. Liang, C. C. Lu, and C. W. Hsieh, "Application of a novel battery charger system to new developed piezoelectric actuator for high speed micropositioning motion," in Proc. ISCAS, 2000, vol. 2, pp. 501-504.

[11] K. Furutani and K. Iida, "Performance of driving method of piezoelectric actuator by using current pulse," in Proc. 9th IEEE Int. Workshop Adv. Motion Cont. , Istanbul, Turkey, 2006, pp. 506-610.

[12] A. J. Fleming and S. O. R. Moheimani, "Precision current and charge amplifiers for driving highly capacitive piezoelectric loads," Electron. Lett., vol. 39, pp. 282-284, 2003.

[13] A. J. Fleming and S. O. R. Moheimani, "Improved current and charge amplifiers for driving piezoelectric loads, and issue in signal processing design for synthesis of shunt damping circuits," J. Intell. Material Syst. and Structures, vol. 15, no. 2, pp. 77-92, Feb. 2004.

[14] T. Higuchi, "Application of electromagnetic impulsive force to precision tools in robot system," in 2nd Int. Symp. Robotics Res., Kyoto, Japan, 1984, vol. 20-23, pp. 144-149.

[15] P. R. Gray, P. J. Hurst, S. H. Lewis , and R. G. Meyer, Analysis and Design of Analog Integrated Circuits, 4th ed. New York: Wiley, 2001, pp. 624-626.

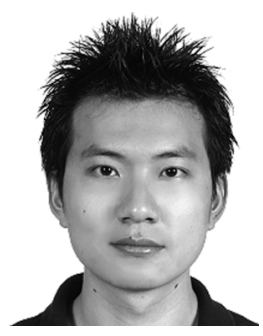

Yung-Cheng Tung was born in Taiwan in 1979. He received the B.S. degree in mechanical engineering from National Chiao-Tung University, Hsinchu, Taiwan, in 2001, and is currently working toward the Ph.D. degree in the Department of Mechanical Engineering, National Chiao Tung University, Taiwan.

His fields of interest include electronic machines, drives, power electronics and mechanical design.

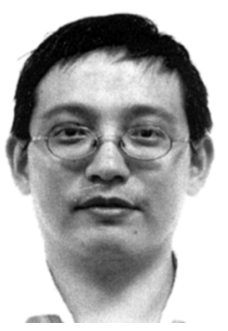

Shyr-Long Jeng was born in Taiwan, in 1965. He received the $\mathrm{Ph}$.D. degree in mechanical engineering at National Chiao-Tung University in 1996. He was appointed an assistant professor in automation engineering at Ta-Hwa Institute of Technology in 1998.

From 1996 to 1998, he joined an electrical motor design company. His current research is in the microprocessor based control and applications.

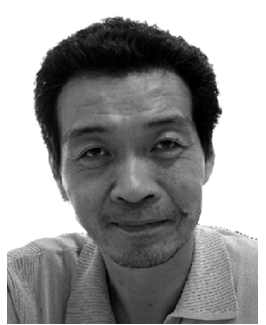

Wei-Hua Chieng was born in Taiwan, in 1959. He received the $\mathrm{Ph} . \mathrm{D}$. degree in mechanical engineering at Columbia University, New York, in 1989.

He has awarded the IBM manufacturing fellowship in 1988 and 1989. He received MSs in electrical and mechanical engineering at Columbia University in 1986 and 1987. During his time at Columbia University his adviser, Prof. David A. Hoeltzel, has brought him into the research in artificial intelligence for mechanical design. He is currently the chairman of mechanical engineering department at National Chiao-Tung University. His research interests include the PC-based controller, flight simulator, and mechatronics devices. 